\title{
Effective Operator Treatment of the Lipkin Model
}

\author{
K.J. Abraham ${ }^{a}$ \& J.P. Vary ${ }^{a}$ \\ ${ }^{a}$ Department of Physics and Astronomy, Iowa State University, Ames, Iowa 50011
}

\begin{abstract}
We analyze the Lipkin Model in the strong coupling limit using effective operator techniques. We present both analytical and numerical results for low energy effective hamiltonians. We investigate the reliability of various approximations used to simplify the nuclear many body problem, such as the cluster approximation. We demonstrate, in explicit examples, certain limits to the validity of the cluster approximation but caution that these limits may be particular to this model where the interactions are of unlimited range.
\end{abstract}


The effective operator method [1] has been used extensively and successfully to obtain the low-lying spectroscopy of complex nuclei [2] with realistic nucleon-nucleon interactions. Central to the methodology of [1] is the construction of a similarity transformation which transforms the original hamiltonian to a new hamiltonian in which a subset of states is decoupled. It is thus possible to construct an effective hamiltonian whose eigenvalues are a subset of the eigenvalues of the full hamiltonian. When this procedure is implemented the effective hamiltonian generally will contain all operators consistent with the symmetries of the bare hamiltonian, even those not present in the original hamiltonian. In particular, for an $N$ fermion problem containing just one and two body operators, the effective hamiltonian can be expected to contain all operators involving up to $N$ fermions consistent with the symmetries of the bare hamiltonian. In the nuclear many body problem, it is customary to make a simplifying approximation such as the cluster approximation [2] where only one and two body operators in the effective hamiltonian are retained. Such approximations are also a feature of schemes attempting to obtain nuclear energy levels starting from a cut-off independent low energy 2 body potential [3]. In order to the test the validity of this approximation it is useful to have a many fermion model which can be exactly solved and in which the cluster approximation can be easily implemented. In what follows, we will construct both analytical and numerical effective hamiltonians for the Lipkin Model [4] and study the effects of neglecting induced operators on the ground state energy.

It is important to note at the outset that due to particular features of the Lipkin Model our implementation of the cluster approximation does not coincide with the truncation practised in [2]. In addition, we emphasize that the cluster approximation makes the most sense when applied to systems of low-density and in our Lipkin model application there is no length scale. Equivalently, the interactions have no limit to their range. For both these reasons, our conclusions concerning the limits of the cluster approximation are restricted to the present application. Nevertheless, the ability to directly investigate the validity limits of the cluster approximation and the dependence on the coupling strength impart significance to these results in the long-standing tradition of testing many-body methods with simple soluble examples.

We adopt the Lipkin Model for the case of $N$ spin 1/2 fermions in an external magnetic field which interact with one another via couplings described by parameters $v$ and $w$. The Hamiltonian may be written as follows

$$
\frac{1}{2} \mu \sum\left(\sigma a_{p \sigma}^{\dagger} a_{p \sigma}\right)+\frac{v}{2} \sum\left(a_{p \sigma}^{\dagger} a_{p^{\prime} \sigma}^{\dagger} a_{p^{\prime}-\sigma} a_{p-\sigma}\right)+\frac{w}{2} \sum\left(a_{p \sigma}^{\dagger} a_{p^{\prime}-\sigma}^{\dagger} a_{p^{\prime} \sigma} a_{p-\sigma}\right)
$$

where the sum runs over the single particle states labelled by $p$ and $p^{\prime}$ and $\sigma$ which takes values \pm 1 . The range of $p$ is taken as $1 \leq p \leq N$. It is customary to introduce quasi-spin operators

$$
\begin{aligned}
J_{+} & =\sum_{p} a_{p,+1}^{\dagger} a_{p,-1} \\
J_{-} & =\sum_{p} a_{p,-1}^{\dagger} a_{p,+1} \\
J_{z} & =\frac{1}{2} \sum_{p \sigma} \sigma a_{p \sigma}^{\dagger} a_{p \sigma}
\end{aligned}
$$


which satisfy the usual angular momentum commutation relations. Hence, we will use the term "angular momentum" to characterize these quasi-spin operators. In terms of the quasi-spin operators the Hamiltonian for the Lipkin Model may be written (following (4) as

$$
H=\mu J_{z}+\frac{1}{2} v\left(J_{+}^{2}+J_{-}^{2}\right)+\frac{1}{2} w\left(J_{+} J_{-}+J_{-} J_{+}\right)
$$

after dropping a term proportional to $w$ which is of no consequence as $w$ will be eventually set to zero. A natural basis for defining the eigenstates of the hamiltonian is the complete set of states of the total angular momentum operator and the $z$ component of the total angular momentum i.e. $\vec{J}=\sum \overrightarrow{j_{p}}$ and $J_{z}=\sum j_{z_{p}}$ with $\overrightarrow{j_{p}}=\frac{1}{2} \overrightarrow{\sigma_{p}}$. In our work, the description in terms of the total angular momentum is equivalent to the description in terms of the quasi-spin. Since $\left[H, J^{2}\right]=0$ we can restrict our attention to multiplets with fixed $j$. We choose the multiplet the largest value of $j$, thus the number of particles is simply twice the largest eigenvalue of $J_{z}$.

For the sake of simplicity we will set $w=0$ and also add to the hamiltonian a constant term $v j(j+1)+\mu j$ for the purpose of making the hamiltonian positive definite, i.e. our hamiltonian in a fixed $j$ multiplet reads

$$
H=\mu\left(J_{z}+J^{2} /(j+1)\right)+\frac{1}{2} v\left(J_{+}^{2}+J_{-}^{2}\right)+v J^{2}
$$

In order to get a feeling for the nature of operators induced in the effective hamiltonian we will consider the case where $\mu=0$ and $j=4$. We label states according to their $J_{z}$ eigenvalues; since there are no terms in the hamiltonian coupling states whose $J_{z}$ eigenvalues are odd to those whose $J_{z}$ eigenvalues are even, we will consider only states whose $J_{z}$ eigenvalues run over even values between -4 to +4 . The hamiltonian is thus five dimensional. Setting $v$ to 1 , the bare hamiltonian (in the basis $-4,4,-2,0,2$ ) takes the form

$$
\left(\begin{array}{ccccc}
20 & 0 & 5.2915 & 0 & 0 \\
0 & 20 & 0 & 0 & 5.2915 \\
5.2915 & 0 & 20 & 9.4648 & 0 \\
0 & 0 & 9.4648 & 20 & 9.4648 \\
0 & 5.2915 & 0 & 9.4648 & 20
\end{array}\right)
$$

Note that with $\mu=0$ the hamiltonian is symmetric under $J_{i} \rightarrow-J_{i}$. Following [1] we define a $P$ Space to contain all states to be treated exactly, and a $Q$ space which is averaged over. We choose the $\mathrm{P}$ Space to span all states with $J_{z}$ eigenvalues running from -2 to +2 and the $Q$ space the remaining two states.

In this basis PHP is

$$
\left(\begin{array}{ccc}
20 & 9.4648 & 0 \\
9.4648 & 20 & 9.4648 \\
0 & 9.4648 & 20
\end{array}\right)
$$

QHQ is

$$
\left(\begin{array}{cc}
20 & 0 \\
0 & 20
\end{array}\right)
$$

and QHP is given by

$$
\left(\begin{array}{ccc}
5.2915 & 0 & 0 \\
0 & 0 & 5.2915
\end{array}\right)
$$


The full hamiltonian may be schematically represented by

$$
\left(\begin{array}{ll}
\text { QHQ } & \text { QHP } \\
\text { PHQ } & \text { PHP }
\end{array}\right)
$$

which is the form which will be used throughout this paper.

In order to arrive at a non-hermitean form of the effective hamiltonian we use the following iteration scheme due to Andreozzi [5]:

$$
\begin{aligned}
X_{0} & =\frac{-1}{Q H Q} Q H P \\
X_{n} & =\frac{1}{q_{n-1}} X_{n-1} p_{n-1} \\
p_{n-1} & =P H P+P H Q \sigma_{n-1} \\
q_{n-1} & =Q H Q-\sigma_{n-1} P H Q
\end{aligned}
$$

$p_{n}$ and $q_{n}$ are the $\mathrm{P}$ and $\mathrm{Q}$ space effective hamiltonians after $n$ iterations, where $n=$ $1,2,3 \ldots \& \sigma_{n-1}=X_{0}+X_{1} \ldots X_{n-1}$. The iterations converge when successive $X_{i}$ become smaller and smaller. Once convergence has been attained $\omega=\sum X_{i}$ is a solution to the Lee Suzuki decoupling equation,

$$
\omega P H Q \omega+\omega P H P-Q H Q \omega-Q H P=0
$$

The iterations defined in Eqs.3-6 are stable and converge smoothly due to the presence of the $v J^{2}$ term added to the Lipkin Model Hamiltonian. The eigenvalues of the non-hermitean effective hamiltonian are in excellent agreement with the lowest three eigenvalues of the full hamiltonian.

In order to analyze the operators induced by the iteration procedure it is necessary to transform the non-hermitean effective hamiltonian to a hermitean form [6]

$$
H_{e f f}=\frac{1}{\sqrt{I_{P}+\omega^{T} \omega}}\left(I_{P}+\omega^{T}\right) H\left(I_{P}+\omega\right) \frac{1}{\sqrt{I_{P}+\omega^{T} \omega}}
$$

In more transparent notation, $H_{\text {eff }}$ can be expressed as

$$
H_{e f f}=\frac{1}{\sqrt{I_{P}+\omega^{T} \omega}}\left(\omega^{T} Q H Q \omega+\omega^{T} Q H P+P H Q \omega+P H P\right) \frac{1}{\sqrt{I_{P}+\omega^{T} \omega}}
$$

This form of the effective hamiltonian arises if the only transformations made are those needed to decouple part of the Hilbert space with no additional unitary transformations on the basis states [7. This prescription leads to the following hermitean effective hamiltonian

$$
\left(\begin{array}{ccc}
12.1103 & 4.4521 & -2.5982 \\
4.4521 & 16.0685 & 4.4521 \\
-2.5982 & 4.4521 & 12.1103
\end{array}\right)
$$

We also evaluated the effective Hamiltonian using a matrix inversion scheme [1, 2] and observe that it produces the same P-space hermitean matrix as the Andreozzi iteration scheme that we use throughout this work.

Comparing the hermitean effective hamiltonian with PHP it is evident that in addition to a global rescaling of the bare matrix elements, operators consistent with the 
symmetries of the bare hamiltonian and the cutoff but not present in the bare hamiltonian have been induced. The hermitean effective hamiltonian, $H_{e f f}$, may also be expressed analytically as

$$
H_{e f f}=\mathrm{P}\left(0.8033 J^{2}-0.9889 J_{z}^{2}+0.2346\left(J_{+}^{2}+J_{-}^{2}\right)-\frac{0.1443}{j(j+1)}\left(J_{+}^{4}+J_{-}^{4}\right)\right) \mathrm{P}
$$

where $\mathrm{P}$ is a projection operator which is included to ensure that $H_{\text {eff }}$ has non-vanishing matrix elements only between $\mathrm{P}$ space states. $\mathrm{P}$ has the form

$$
\frac{J^{2}}{j(j+1)}+\gamma\left(J_{+}^{8} J_{-}^{8}+J_{-}^{8} J_{+}^{8}\right)
$$

The constant $\gamma$ is given by $-\left(<-4\left|J_{-}^{8}\right| 4><-4\left|J_{+}^{8}\right| 4>\right)^{-1}$. It is worth pointing out that in addition to new off diagonal operators, diagonal operators not present in Eq. [6] appear in Eq. 14. Note that $<-2\left|H_{\text {eff }}\right|-2>=<2\left|H_{\text {eff }}\right| 2>$, which rules out the possibility of Eq. 14 containing an induced diagonal operator of the form $J_{z}^{m}(m$ odd), which in any case would not have been expected on symmetry grounds.

The new diagonal operators have the same effect as a state dependent rescaling of the diagonal matrix elements of the bare hamiltonian; a similar feature arises in the nuclear many body problem. In the cluster approximation, the matrix elements of the effective hamiltonian are typically constrained to be related to those of the bare hamiltonian by channel dependent renormalization. To implement the analog of the cluster approximation would require setting $<2\left|H_{\text {eff }}\right|-2>=<-2\left|H_{\text {eff }}\right|+2>=0$ or equivalently dropping the $J_{ \pm}^{4}$ terms in Eq. 14. Since the dimension of the effective hamiltonian is rather small there are just two matrix elements to be omitted in this two body cluster approximation. With a larger value of $j$, or equivalently a larger number of fermions, the number of matrix elements omitted in the cluster approximation will be larger. The rest of this paper is devoted to a detailed numerical investigation of the validity of the cluster approximation in the Lipkin Model with larger values of $j$ than we have hitherto considered.

We next consider the case where $j=8, \mu=0.1$ and $v=0.03$. With these parameters the dimensionless quantity $(v j) / \mu$ is 2.4. Thus we are in a regime where perturbation theory cannot necessarily be trusted. The Q-Space is chosen to be one dimensional, containing the state whose $J_{z}$ eigenvalue is +8 . All remaining states are absorbed into the $\mathrm{P}$-space which is 8 dimensional as we once again keep only states whose $J_{z}$ eigenvalues are even. Choosing the basis for $\mathrm{H}$ as $8,6,4,2,0,-2,-4,-6,-8 \mathrm{PHP}$ is given by

$$
\left(\begin{array}{cccccccc}
3.5600 & 0.7010 & 0 & 0 & 0 & 0 & 0 & 0 \\
0.7010 & 3.3600 & 0.9439 & 0 & 0 & 0 & 0 & 0 \\
0 & 0.9439 & 3.1600 & 1.0649 & 0 & 0 & 0 & 0 \\
0 & 0 & 1.0649 & 2.9600 & 1.0649 & 0 & 0 & 0 \\
0 & 0 & 0 & 1.0649 & 2.7600 & 0.9439 & 0 & 0 \\
0 & 0 & 0 & 0 & 0.9439 & 2.5600 & 0.7010 & 0 \\
0 & 0 & 0 & 0 & 0 & 0.7010 & 2.3600 & 0.3286 \\
0 & 0 & 0 & 0 & 0 & 0 & 0.3286 & 2.1600
\end{array}\right)
$$

and QHP is given by

$$
\left(\begin{array}{llllllll}
0.3286 & 0 & 0 & 0 & 0 & 0 & 0 & 0
\end{array}\right)
$$


QHQ is simply $<8|H| 8>$ or 3.7600 Once again the iterative scheme defined earlier is implemented leading to the following hermitean effective hamiltonian, $H_{e f f}$

$$
\left(\begin{array}{cccccccc}
\mathbf{3 . 3 1 5 9} & \mathbf{0 . 4 2 5 1} & -0.3246 & -0.2780 & -0.1751 & -0.0782 & -0.0022 & -0.0027 \\
\mathbf{0 . 4 2 5 1} & \mathbf{3 . 1 3 6 0} & \mathbf{0 . 6 8 0 5} & -0.2254 & -0.1418 & -0.0633 & -0.0180 & -0.0022 \\
-0.3246 & \mathbf{0 . 6 8 0 5} & \mathbf{2 . 8 5 0 1} & \mathbf{0 . 7 9 9 7} & -0.1668 & -0.0745 & -0.0211 & -0.0026 \\
-0.2780 & -0.2254 & \mathbf{0 . 7 9 9 7} & \mathbf{2 . 7 3 3 1} & \mathbf{0 . 9 2 2 1} & -0.0637 & -0.0181 & -0.0022 \\
-0.1751 & -0.1418 & -0.1668 & \mathbf{0 . 9 2 2 1} & \mathbf{2 . 6 7 0 2} & \mathbf{0 . 9 0 3 8} & -0.0114 & -0.0014 \\
-0.0782 & -0.0633 & -0.0745 & -0.0637 & \mathbf{0 . 9 0 3 8} & \mathbf{2 . 5 4 2 1} & \mathbf{0 . 6 9 5 9} & -0.0006 \\
-0.0222 & -0.0180 & -0.0211 & -0.0181 & -0.0114 & \mathbf{0 . 6 9 5 9} & \mathbf{2 . 3 5 8 6} & \mathbf{0 . 3 2 8 5} \\
-0.0027 & -0.0022 & -0.0026 & -0.0022 & -0.0014 & -0.0006 & \mathbf{0 . 3 2 8 5} & \mathbf{2 . 1 6 0 0}
\end{array}\right)
$$

Comparing the $H_{\text {eff }}$ with PHP we see immediately that new operators have been induced, the induced transitions corresponding to $\left|\Delta J_{z}\right|=4,6, \ldots 14$. The lowest eigenvalue of the effective hamiltonian is 1.0460 which is in excellent agreement with the lowest eigenvalue of the full hamiltonian.

It is noteworthy that the magnitude of matrix elements of the induced operators is generally smaller than those of the operators present in the bare hamiltonian despite the fact that the interaction has for all intents and purposes infinite range. This will be a recurring feature of the effective hamiltonians we construct and suggests that in hamiltonians with a finite range interaction, such as the nuclear many body problem, the relative size of induced many body operators may indeed be small.

Implementing the 2 body cluster approximation corresponds to retaining all matrix elements of $H_{\text {eff }}$ which are in bold face and setting all other matrix elements to 0 . The lowest eigenvalue of the hamiltonian which results is 1.1238 , thus the effect of the cluster approximation is not too drastic. In order to offset the possible effect of the shift in the ground state energy, we consider the splitting between the ground and first excited state as is done in the treatment of the nuclear many body problem [2]. With no cluster truncation of $H_{\text {eff }}$, we obtain 0.5974 . With the cluster truncation we get 0.5769 ; the effects of the cluster truncation are apparently comparable in size and sign for both the ground and first excited states. This might have been anticipated on perturbative grounds alone, as the state left out of the $\mathrm{P}$ space has the highest unperturbed energy and thus might be expected to have a minimal effect on the true ground state energy.

As a check on this line of reasoning we consider the case where the Q space consists just of the state whose $J_{z}$ eigenvalue is 0 . Since the unperturbed energy of the omitted state is not as high as before, the cluster truncation estimate for the ground state energy can be expected to be less accurate than before. Furthermore, with this choice of Q space we can address the efficacy of the cluster approximation when intruder states are present. Strictly speaking intruder states arise when the perturbed energies of Q space states lie among the perturbed energies of the $\mathrm{P}$ space states. With our new choice of $\mathrm{Q}$ space, the unperturbed energies of the Q space states lie among the unperturbed energies of the $\mathrm{P}$ space states, simulating the effect of intruder states. Apart from the change in the choice of $\mathrm{Q}$ space, all other parameters retain the same values as before. Choosing 
the basis for $\mathrm{H}$ as $0,-8,-6,-4,-2,2,4,6,8 \mathrm{PHP}$ is given by

$$
\left(\begin{array}{cccccccc}
2.1600 & 0.3286 & 0 & 0 & 0 & 0 & 0 & 0 \\
0.3286 & 2.3600 & 0.7010 & 0 & 0 & 0 & 0 & 0 \\
0 & 0.7010 & 2.5600 & 0.9439 & 0 & 0 & 0 & 0 \\
0 & 0 & 0.9439 & 2.7600 & 0 & 0 & 0 & 0 \\
0 & 0 & 0 & 0 & 3.1600 & 0.9439 & 0 & 0 \\
0 & 0 & 0 & 0 & 0.9439 & 3.3600 & 0.7010 & 0 \\
0 & 0 & 0 & 0 & 0 & 0.7010 & 3.5600 & 0.3286 \\
0 & 0 & 0 & 0 & 0 & 0 & 0.3286 & 3.7600
\end{array}\right)
$$

In the same basis QHP is given by

$$
\left(\begin{array}{llllllll}
0 & 0 & 0 & 1.0649 & 1.0649 & 0 & 0 & 0
\end{array}\right)
$$

and QHQ is just $<0|H| 0>$ or 2.9600. Repeating the same iterative procedure yields the following $H_{e f f}$,

$$
\left(\begin{array}{cccccccc}
\mathbf{2 . 1 6 0 0} & \mathbf{0 . 3 2 8 5} & -0.0005 & -0.0052 & -0.0063 & -0.0020 & -0.0011 & -0.0003 \\
\mathbf{0 . 3 2 8 5} & \mathbf{2 . 3 5 8 8} & \mathbf{0 . 6 9 6 6} & -0.0403 & -0.0490 & -0.0158 & -0.0092 & -0.0027 \\
-0.0005 & \mathbf{0 . 6 9 6 6} & \mathbf{2 . 5 4 4 3} & \mathbf{0 . 8 0 7 2} & -0.1675 & -0.0564 & -0.0328 & -0.0097 \\
-0.0052 & -0.0403 & \mathbf{0 . 8 0 7 2} & \mathbf{2 . 2 3 9 9} & -0.7796 & -0.4809 & -0.2771 & -0.0792 \\
-0.0063 & -0.0490 & -0.1675 & -0.7796 & \mathbf{2 . 0 6 0 6} & \mathbf{0 . 3 5 2 4} & -0.3415 & -0.0981 \\
-0.0020 & -0.0158 & -0.0564 & -0.4809 & \mathbf{0 . 3 5 2 4} & \mathbf{3 . 1 5 7 1} & \mathbf{0 . 5 8 3 0} & -0.0347 \\
-0.0011 & -0.0092 & -0.0328 & -0.2771 & -0.3415 & \mathbf{0 . 5 8 3 0} & \mathbf{3 . 4 9 1 4} & \mathbf{0 . 3 0 8 5} \\
-0.0003 & -0.0027 & -0.0097 & -0.0792 & -0.0981 & -0.0347 & \mathbf{0 . 3 0 8 5} & \mathbf{3 . 7 5 4 1}
\end{array}\right)
$$

Once again, the lowest eigenvalue of $H_{\text {eff }}$ coincides with that of the full Hamiltonian to 5 significant figures. In order to implement the two body cluster approximation, we retain only the bold faced matrix elements of $H_{\text {eff }}$, setting all other matrix elements to 0 . The lowest eigenvalue of the resulting hamiltonian overshoots the true ground state by $25 \%$. On the other hand, the splitting between the first excited state and the ground state with the cluster truncation is 0.6228 , compared with the true value of 0.5974 . Thus, the accuracy of the cluster approximation depends on the choice of the observable: the absolute eigenvalue or the eigenvalue spacing.

As a further check we now consider a situation where i.e. QHQ $=<-8|\mathrm{H}|-8>$ keeping all the coupling constants the same. The unperturbed $\mathrm{Q}$ space energy is smallest among all the cases we have considered; the effect of the cluster truncation on the ground state energy may be expected to be the largest. In the basis in which the Hamiltonian is ordered -8,-6,-4,-2,0,2,4,6,8 PHP takes the form

$$
\left(\begin{array}{cccccccc}
2.3600 & 0.7010 & 0 & 0 & 0 & 0 & 0 & 0 \\
0.7010 & 2.5600 & 0.9439 & 0 & 0 & 0 & 0 & 0 \\
0 & 0.9439 & 2.7600 & 1.0469 & 0 & 0 & 0 & 0 \\
0 & 0 & 1.0469 & 2.9600 & 1.0649 & 0 & 0 & 0 \\
0 & 0 & 0 & 1.0649 & 3.1600 & 0.9439 & 0 & 0 \\
0 & 0 & 0 & 0 & 0.9439 & 3.3600 & 0.7010 & 0 \\
0 & 0 & 0 & 0 & 0 & 0.7010 & 3.5600 & 0.3286 \\
0 & 0 & 0 & 0 & 0 & 0 & 0.3286 & 3.7600
\end{array}\right)
$$


and QHQ is given by

$$
\left(\begin{array}{llllllll}
0.3286 & 0 & 0 & 0 & 0 & 0 & 0 & 0
\end{array}\right)
$$

In exactly the same manner as before, we compute the effective hamiltonian and obtain

$$
\left(\begin{array}{cccccccc}
\mathbf{2 . 3 3 1 5} & \mathbf{0 . 6 4 3 4} & -0.1302 & -0.2085 & -0.2453 & -0.2098 & -0.1215 & -0.0360 \\
\mathbf{0 . 6 4 3 4} & \mathbf{2 . 5 1 1 1} & \mathbf{0 . 8 3 3 6} & -0.1764 & -0.2072 & -0.1771 & -0.1025 & -0.0304 \\
-0.1302 & \mathbf{0 . 8 3 3 6} & \mathbf{2 . 5 1 1 2} & \mathbf{0 . 6 6 7 1} & -0.4672 & -0.3993 & -0.2311 & -0.0686 \\
-0.2085 & -0.1764 & \mathbf{0 . 6 6 7 1} & \mathbf{2 . 3 2 4 0} & \mathbf{0 . 3 1 7 9} & -0.6383 & -0.3695 & -0.1096 \\
-0.2453 & -0.2072 & -0.4672 & \mathbf{0 . 3 1 7 9} & \mathbf{2 . 2 8 2 6} & \mathbf{0 . 1 9 4 2} & -0.4340 & -0.1288 \\
-0.2098 & -0.1771 & -0.3993 & -0.6383 & \mathbf{0 . 1 9 4 2} & \mathbf{2 . 7 1 9 3} & \mathbf{0 . 3 3 0 1} & -0.1100 \\
-0.1215 & -0.1025 & -0.2311 & -0.3695 & -0.4340 & \mathbf{0 . 3 3 0 1} & \mathbf{3 . 3 4 5 3} & \mathbf{0 . 2 6 4 9} \\
-0.0360 & -0.0304 & -0.0686 & -0.1096 & -0.1288 & -0.1100 & \mathbf{0 . 2 6 4 9} & \mathbf{3 . 7 4 1 1}
\end{array}\right)
$$

Retaining just the bold faced terms in the two body cluster approximation leads to a 20 $\%$ error in the estimate of the ground state energy. Furthermore, the splitting between the first excited state and the ground state is now 0.6293, compared with the true value of 0.5974. As expected the error is substantial but has actually decreased instead of increasing as we originally anticipated on the basis of perturbative arguments. Our results are summarised in the table below: $\left(E_{g s}\right.$ is the ground state energy and 0.5974 is the true splitting between the ground state and the first excited state)

\begin{tabular}{|l|l|l|l|l|}
\hline Q Space $J_{z}$ & $<Q|H| Q>$ & $E_{g s}\left(H_{e f f}\right)$ & $E_{g s}$ (Cluster) & Splitting \\
\hline 8 & 3.7600 & 1.0460 & 1.1238 & $0.5769(0.5974)$ \\
\hline 0 & 2.9600 & 1.0460 & 1.3130 & $0.6228(0.5974)$ \\
\hline-8 & 2.1600 & 1.0460 & 1.2547 & $0.6293(0.5974)$ \\
\hline
\end{tabular}

Comparing the three cases we may conclude that even when the dimensions of the $\mathrm{P}$ and $\mathrm{Q}$ spaces are fixed, the accuracy of the cluster approximation in fixing the ground state is strongly affected by the choice of $\mathrm{P}$ and $\mathrm{Q}$ spaces in a manner which cannot be anticipated by perturbation theory alone. The splitting between the first excited state and the ground state is rather less sensitive to the choice of $\mathrm{P}$ space. As might be expected, larger cluster sizes can improve the estimate of the ground state energy. In the case where the Q Space contains $<-8|H|-8>$, going from 2 body to 4 body clusters reduces the discrepancy in the ground state energy from $20 \%$ to $15 \%$ which is an appreciable but not spectacular improvement.

We now investigate what happens when the size of the $\mathrm{P}$ space relative to the full Hilbert space shrinks; the Q Space is spanned by states whose eigenvalues under $J_{z}$ are $8,6,-6, \& 8$ leaving a 5 dimensional $\mathrm{P}$ space. If the basis for the Hamiltonian is ordered $8,6,-6,-8,-4,-2,0,2,4$ QHQ is given by

$$
\left(\begin{array}{cccc}
3.7600 & 0.3268 & 0 & 0 \\
0.3286 & 3.5600 & 0 & 0 \\
0 & 0 & 2.3600 & 0.3286 \\
0 & 0 & 0.3286 & 2.1600
\end{array}\right)
$$


PHP is given by

$$
\left(\begin{array}{ccccc}
2.5600 & 0.9439 & 0 & 0 & 0 \\
0.9439 & 2.7600 & 1.0649 & 0 & 0 \\
0 & 1.0649 & 2.9600 & 1.0649 & 0 \\
0 & 0 & 1.0649 & 3.1600 & 0.9439 \\
0 & 0 & 0 & 0.9439 & 3.3600
\end{array}\right)
$$

and QHP by

$$
\left(\begin{array}{ccccc}
0 & 0 & 0 & 0 & 0 \\
0 & 0 & 0 & 0 & 0.7010 \\
0.7010 & 0 & 0 & 0 & 0 \\
0 & 0 & 0 & 0 & 0
\end{array}\right)
$$

Once again we construct the effective Hamiltonian by iteration and $H_{\text {eff }}$ is obtained to be

$$
\left(\begin{array}{ccccc}
\mathbf{1 . 5 6 8 8} & \mathbf{0 . 1 6 9 2} & -0.4703 & -0.0242 & 0.0993 \\
\mathbf{0 . 1 6 9 2} & \mathbf{2 . 0 7 0 3} & \mathbf{0 . 3 3 9 7} & -0.4884 & -0.1198 \\
-0.4703 & \mathbf{0 . 3 3 9 7} & \mathbf{1 . 9 5 3 9} & \mathbf{0 . 1 5 0 2} & -0.3723 \\
-0.0242 & -0.4884 & \mathbf{0 . 1 5 0 2} & \mathbf{2 . 1 4 4 9} & \mathbf{0 . 3 0 6 3} \\
0.0993 & -0.1198 & -0.3723 & \mathbf{0 . 3 0 6 3} & \mathbf{2 . 4 3 3 0}
\end{array}\right)
$$

Once again, the lowest eigenvalue of $H_{\text {eff }}$ and that of the full hamiltonian agree to 5 significant figures. Implementing the cluster approximation amounts to retaining only the bold faced terms in $H_{e f f}$. Doing so yields a truncated effective Hamiltonian whose lowest eigenvalue overestimates the true ground state energy by over $41 \%$. In addition, the splitting between the ground state energy and the first excited state is 0.2134 , drastically different from the true value of 0.5974 . This suggests that the accuracy of the two body cluster approximation decreases as the size of the $\mathrm{P}$ space relative to the full Hilbert space decreases.

Finally, we investigate what happens when the coupling strength $v$ which was hitherto fixed to be 0.03 is allowed to vary. We go back to the case where the cluster approximation gave the best results, i.e. with $\mathrm{QHQ}=<8|\mathrm{H}| 8>$, but now set $v$ to be 0.1 . Once again, $H_{\text {eff }}$ with no cluster truncation reproduces the true ground state energy to five significant figures. However, if the two body cluster approximation is implemented on $H_{e f f}$, there is a $39 \%$ error in the ground state energy instead of just under $8 \%$ with the original value of $v$. In addition the splitting between the ground state and the first excited state is poorly reproduced in the cluster approximation.

As expected, the accuracy of the two body cluster approximation decreases at large couplings.

To conclude, we have investigated the relevance of induced operators which arise in an effective operator treatment of the Lipkin Model. Ignoring these operators as is done in the cluster approximation, leads to errors of varying magnitude but which can be uncomfortably large. The errors from the cluster approximation in these soluble examples are likely to be larger than those in more realistic applications due in part due to the infinite range of the interaction in the Lipkin Model. In hamiltonians where the interaction is of finite range, such as the nuclear many body problem the errors may not be as large. Nonetheless, even with infinite range interactions, it is possible to establish guidelines for minimising the error which may well be relevant for a broader class of interactions. Based on our numerical results, the approximation seems to work best for couplings which are not too large and when the model space states constitute 
as large a fraction as possible of the full Hilbert space. In other instances the errors can be substantial even when the couplings are only moderately large. We note that more realistic applications typically investigate carefully the convergence rate of the eigenvalues, at fixed cluster size, with increasing $\mathrm{P}$-space dimensions. In this way, one anticipates that the errors due to cluster truncation are minimized.

This work was supported in part by U.S. DOE Grant No. DE-FG-02-87ER-40371, Division of High Energy and Nuclear Physics.

\section{References}

[1] K. Suzuki \& S.Y. Lee; Prog. Theor. Phys. 64, 2091(1980)

[2] P. Navratil, J.P. Vary \& B.R.Barrett; Phys. Rev. Lett. 84, 5728(2000); Phys.Rev C 62, 054311(2000); P. Navratil, J.P. Vary, W.E. Ormand and B.R. Barrett, Phys. Rev. Lett. 87, 172502(2001)

[3] Scott Bogner, T.T.S. Kuo, L.Coraggio, A Covello, \& N.Itaco;

Phys.Rev.C 65, 051301, 2002

S.K. Bogner \& T.T.S Kuo; Phys.Lett.B 500 279, 2001.

[4] H.J. Lipkin,N. Meshkov, \& A.J.Glick; Nucl. Phys. 62,188 (1965)

[5] Francesco Andreozzi; Phys.Rev.C 54684 (1996)

[6] K.G. Wilson; Phys.Rev.D 21438 (1970)

K. Suzuki \& R. Okamoto; Prog.Theor.Phys. 92, 1045(1994)

[7] K. Suzuki;Prog.Theor.Phys. 68, 246(1982); 